\title{
MACROPHAGE ACTIVATION SYNDROME AS THE FIRST CLINICAL MANIFESTATION OF SYSTEMIC LUPUS ERYTHEMATOSUS - CASE REPORT
}

Sean Hideo Shirata Lanças (Faculdade de Medicina de Botucatu, Botucatu, SP, Brasil), Matheus Zanata Brufatto (Faculdade de Medicina de Botucatu, Botucatu, SP, Brasil), Lais De Vico (Faculdade de Medicina de Botucatu, Botucatu, SP, Brasil), João Paulo Canaan (Faculdade de Medicina de Botucatu, Botucatu, SP, Brasil), Luiz Eduardo Valente (Faculdade de Medicina de Botucatu, Botucatu, SP, Brasil), Henrique Pereira Sampaio (Faculdade de Medicina de Botucatu, Botucatu, SP, Brasil), Andrea Almeida Peduti Batista (Faculdade de Medicina de Botucatu, Botucatu, SP, Brasil), Fabio Vicente Leite (Faculdade de Medicina de Botucatu, Botucatu, SP, Brasil), Sula Glaucia Lage Drumond (Faculdade de Medicina de Botucatu, Botucatu, SP, Brasil)

\section{BACKGROUND}

Macrophage activation syndrome comprises a rare e extremely severe condition, characterized by an anomalous hyperactivation of macrophages, with consequent phagocytosis of hematopoietic cells, besides an uncontrolled hyperinflammatory systemic response.

\section{CASE REPORT}

A 30-year-old female, caucasian, presented to our service with a prior history of 42 days of fever of unknown origin, alongside with dry cough, dyspnea e a palpable purpura in lower limbs. Evolved with a generalized tonic-clonic seizure followed by a cardiac arrest, promptly reverted. She also presented with elevated inflammatory markers, pancytopenia, worsening of renal function, proteinuria, hematuria and granular cylinders in the urine, besides elevated triglycerides and ferritin, and decreased fibrinogen. We also detected signs of pneumonitis, pleuritis, pleural and pericardial effusion, confirmed by image assessment. Bone marrow biopsies evidenced multiple activated macrophages and hemophagocytosis of mature elements, leading to a diagnosis of Macrophage Activation Syndrome. Excluded infectious disorders, immunosuppressive treatment was initiated firstly with Etoposide $150 \mathrm{mg} / \mathrm{m} 2$ plus Dexamethasone $10 \mathrm{mg} / \mathrm{m} 2 /$ day by the Hematology. Further investigation ruled out other chronic infectious and malignant etiologies, presenting with a positive ANA and Coombs, a diagnosis of Systemic Lupus Erythematosus was made, and the treatment scheme was changed for methylprednisolone $1 \mathrm{~g} / \mathrm{day}$ for 3 days, followed by pulse-therapy with cyclophosphamide $(0,6 \mathrm{~g} / \mathrm{m} 2)$. She evolved with gradual control of hematologic activity, however presented a medullary aplasia with the need of repeated transfusion support, besides other complications such as febrile neutropenia and grade 2 mucositis. Despite complications, she was extubated without any neurologic sequels. One day after the infusion of the Cyclophosphamide, began with dyspnea and systemic congestion, compatible with an acute cardiac insuffiency, with a reduced ejection fraction and global hypokinesis by the Echocardiogram (Ventricular ejection fraction of $24 \%$ ). Clinical management of the cardiac decompensation was initiated, and human immunoglobulin ( $2 \mathrm{~g} / \mathrm{Kg}$ on 5 days) was indicated for the lupus myocarditis as a rescue therapy. There was great clinical improvement, and a new control Echocardiogram showed complete restoration of the systolic function. On the following days, the patient evolved with global improvement, completing antibiotics, and was put on monthly pulses of cyclophosphamide and tapering corticosteroids.

\section{CONCLUSION}

Macrophage activation syndrome comprehends a severe and less frequently recognized condition that can present as a secondary complication from rheumatologic diseases such as Lupus, being its recognition fundamental for quickly diagnosis and prompt treatment, seeking the goals of better outcomes. 